\title{
Evaluation of the Conspicuousness of Emergency Exit Signs
}

\author{
TADAHISA JIN, TOKIYOSHI YAMADA \\ Fire Research Institute \\ 3-14-1 Nakahara, Mlitaka-shi \\ Tokyo 181, Japan
}

SADAO TAKAHASHI

Toshiba Electric Equipment Corp.

1-201-1 Funakosi-cho, Yokosuka 237, Japan

\author{
SATORU KAWAI \\ Chukyo University \\ 101-2 Yagotohonmachi, Showa-ku \\ Nagoya 466, Japan
}

\begin{abstract}
An experimental study was carried out to determine the conspicuousness of the three sizes of the emergency exit sign in an underground shopping plaza where many lights of high luminance existed. The research shows noticeability is not only dependent of the size of the emergency exit sign, but on its luminance. We also measured the visual impact of self-flashing type emergency exit sign and compared with that of a control one. The results showed that the conspicuousness of the self-flashing signs were remarkably good in a background with many distractions from other light sources.
\end{abstract}

KEYWORDS: Conspicuousness, Emergency Exit Sign, Self-Flashing Sign, Evacuation, Visibility.

\section{INTRODUCTION}

An emergency exit sign, which indicates a location and/or direction of emergency exit and leads evacuees to a safety place swiftly, is important in case of fire or other emergency. In Japan, we are currently using three sizes of emergency exit sign. However, the conspicuousness of the exit sign in a background with many other light sources was not known.

In the first experiment, we measured the conspicuousness of the ordinary type exit sign in an underground shopping plaza in business hours. Here experimental variables were the observation distance, size and luminance of the exit sign. In the second experiment, the conspicuousness of self-flashing type exit sign (flashing the lamp in the sign) was compared with that of ordinary type exit sign.

\section{EXPERIMENT}

An emergency exit sign currently used in Japan is shown in Photo. 1. The luminance of exit sign used in this experiment is shown in Table 1. The plaza has a long straight shopping mall of $5.8 \mathrm{~m}$ width, 3.0 $\mathrm{m}$ height. The general appearance of the mall and luminance of light noises around the exit sign are 
shown in Photo. 2. An exit sign was fixed to a ceiling in one side of the wall. The bottom of sign was $2.5 \mathrm{~m}$ high above the floor.

The subjects were required to walk towards the sign and to evaluate conspicuousness of an exit sign at distances of $60 \mathrm{~m}, 50 \mathrm{~m}, 40 \mathrm{~m}, 30 \mathrm{~m}, 20 \mathrm{~m}$ and $10 \mathrm{~m}$ from it. Thirty three subjects participated in this experiment and each subject had good eye sight and had no defects of vision. The age and visual acuity of each subject are shown in Table 2. The evaluation was reported by checking the five successive categories shown in Table 3.

The aim of the second experiment was to compare conspicuousness of the self-flashing type exit sign with that of ordinary type one. A flashing type exit sign was installed in one side of the mall and ordinary type sign was set on the other side. Observing spots were the same as ones in the first experiment. The only experimental variable is the observation distance from the sign. Successive five evaluation categories used in this experiment are shown in Table 4.

\section{CONSPICUOUSNESS OF ORDINARY TYPE EXIT SIGN}

Figure 1 shows the relation between the conspicuousness of the ordinary type exit sign and the observing distance from exit sign. The conspicuousness decreases with increasing the distance from exit sign. The large size exit sign

TABLE 1. Surface luminance of ordinary exit sign used in this paper.

\begin{tabular}{lrr}
\hline Size & White Part & Green Part \\
\hline Large & $986 \mathrm{~cd} / \mathrm{m}^{2}$ & $192 \mathrm{~cd} / \mathrm{m}^{2}$ \\
Medium & 785 & 129 \\
Smal1 & 325 & 35 \\
\hline
\end{tabular}

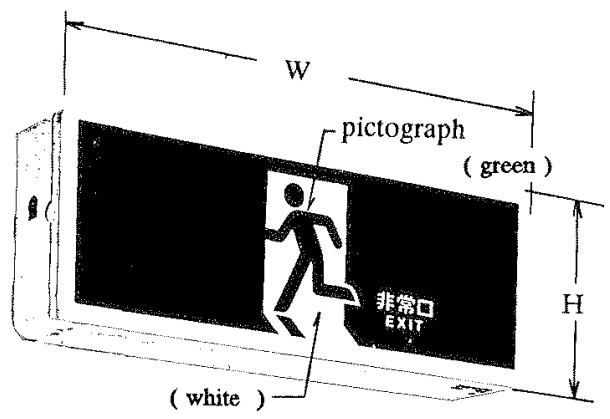

Photo.1. Emergency exit sign currently used in Japan : Sizes $($ large $=h 40 \times w 120 \mathrm{~cm}$, medium $=h 20 \times w 60$ $\mathrm{cm}, \mathrm{small}=\mathrm{h} 13 \times \mathrm{w} 36 \mathrm{~cm}$ )

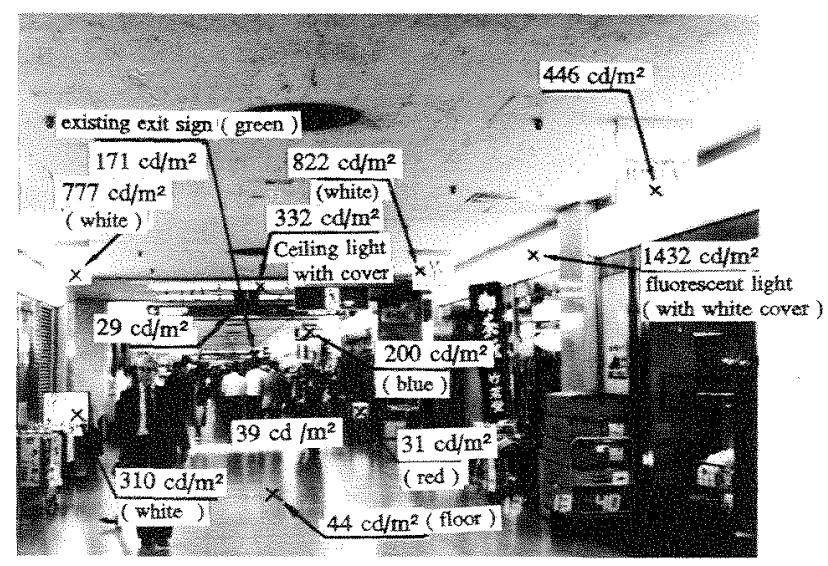

PHOTO.2. Outlook of the mall and luminance distribution around the exit sign. 
is more conspicuous than the small size one. From the figure, it may be said that the distance at which the exit sign can be understood is $80 \mathrm{~m}$ for large size sign, $30 \mathrm{~m}$ for medium one, and only $8 \mathrm{~m}$ for small one.

Figure 2 shows the relation between conspicuousness of the ordinary type exit sign and luminance of the sign at $30 \mathrm{~m}$ distance from the exit sign. This indicates that the conspicuousness increases with increasing luminance of the sign. Therefore, the conspicuousness of the exit sign depends not only on size of the sign, but on its luminance.

TABLE 2. Age and visual acuity of subjects

\begin{tabular}{|c|c|c|c|c|c|c|c|c|c|c|c|}
\hline No. & Age & Sex & $\begin{array}{l}\text { Visual } \\
\text { acuity }\end{array}$ & No. & Age & Sex & $\begin{array}{l}\text { Visual } \\
\text { acuity }\end{array}$ & No. & Age & Sex & $\begin{array}{l}\text { Visual } \\
\text { acuity }\end{array}$ \\
\hline 1. & 37 & $\mathrm{M}$ & 1.5 & 12. & 27 & $M$ & $* * *$ & 23. & 33 & $\mathrm{M}$ & 1.2 \\
\hline 2. & 39 & $M$ & $* * *$ & 13. & 23 & $M$ & 1.0 & 24. & 45 & $M$ & 1.5 \\
\hline 3. & 32 & $\mathrm{M}$ & 0.8 & 14. & 40 & $M$ & 1.5 & 25. & 60 & $\mathrm{M}$ & 1.0 \\
\hline 4. & 34 & $\mathrm{M}$ & 1.5 & 15. & 19 & $\mathrm{M}$ & $* * *$ & 26. & 37 & $\mathrm{M}$ & 1.0 \\
\hline 5. & 60 & $\mathrm{M}$ & 1.2 & 16. & 31 & $M$ & 0.5 & 27. & 36 & $\mathrm{M}$ & $* * * x$ \\
\hline 6. & 30 & $\mathrm{M}$ & 0.7 & 17. & 21 & $F$ & 2.0 & 28 & 24 & $M$ & 1.5 \\
\hline 7. & 40 & $M$ & 1.2 & 18. & 48 & $M$ & 1.0 & 29. & 42 & $M$ & $x * x$ \\
\hline 8. & 39 & $\mathrm{M}$ & 1.0 & 19. & 36 & $M$ & 1.0 & 30. & 21 & $F$ & 1.2 \\
\hline 9 & 23 & $\mathrm{M}$ & 1.2 & 20 & 24 & $\mathrm{M}$ & 0.8 & 31. & 23 & $\mathrm{M}$ & 1.0 \\
\hline 10. & 43 & $\mathrm{M}$ & 1.0 & 21 . & 39 & M & 1.2 & 32. & 22 & $\mathrm{~F}$ & 1.0 \\
\hline 11. & 21 & $\mathbf{F}$ & 1.0 & 22. & 46 & $\mathrm{~F}$ & 1.0 & 33. & 21 & $M$ & 1.5 \\
\hline
\end{tabular}

(cf. M:male, F:Female, $* * * *:$ unknown)

TABLE 3. Categories for evaluating conspicuousness of ordinary type exit sign.

\begin{tabular}{lll}
\hline & evaluation categories & (marks in Fig.) \\
\hline (1) The exit sign is fairly conspicuous. & $(++)$ \\
(2) The exit sign is slightly conspicuous. & $(+)$ \\
(3) The exit sign is similar to the general level. & (general) \\
(4) The exit sign is less conspicuous. & $(-)$ \\
(5) The exit sign is not conspicuous at al1. & $(-)$ \\
\hline
\end{tabular}

TABLE 4. Categories for evaluating conspicuousness of self-flashing type exit sign.

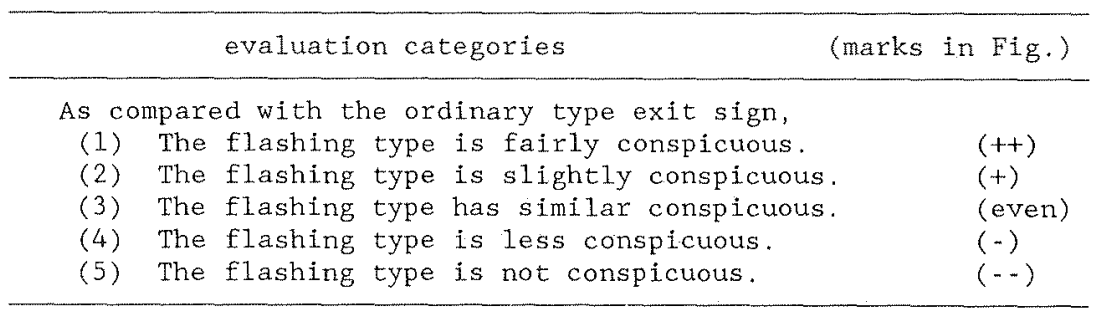




\section{DISCUSSION}

\section{Visibility and Conspicuousness of Exit Sign}

Prior to this experimental study, another experimental study on visibility of exit sign had been done. In that experiment, the original type exit sign was observed in a background without other light sources. Figure 3 shows one of the results concerned with the relationship between a visibility expressed by visual angle and a surface luminance of ordinary exit sign.[1]

In this figure visibility is represented on two discrimination levels; one is the level in which person can distinguisn the details of a pictograph and the other is the level in which only a direction of the running person (pictograph) is distinguishable.

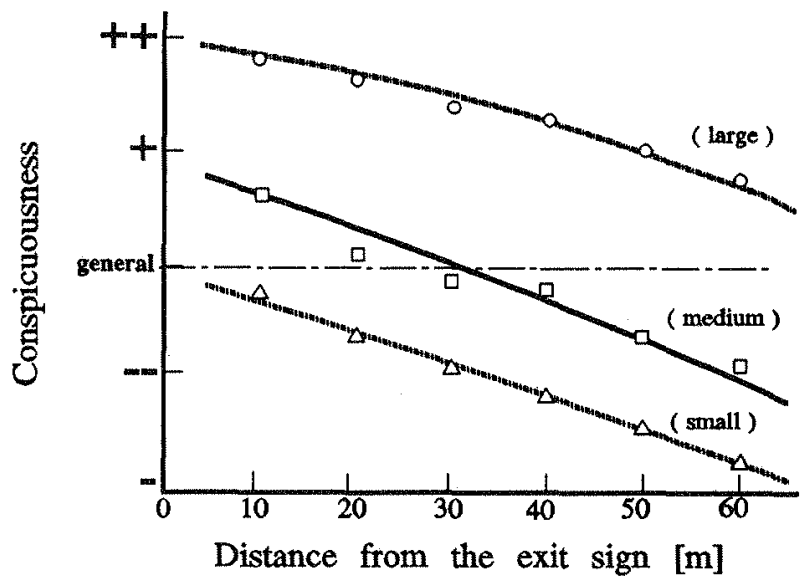

FIGURE 1. Relation between conspicuousness of ordinary type exit sign and distance from the sign.

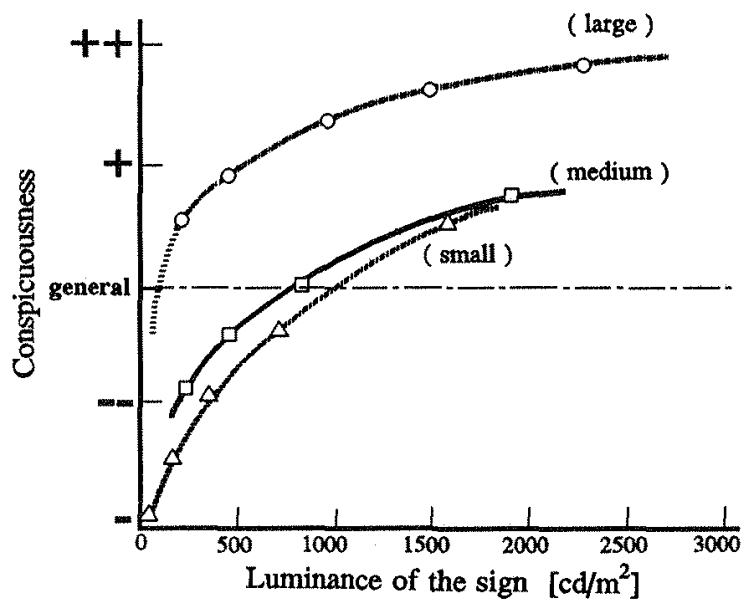

FIGURE 2. Relation between conspicuousness of ordinary type exit sign and surface luminance of the sign. 
This result indicates that visibility is almost constant when luminance of white part of the exit sign is over $300 \mathrm{~cd} / \mathrm{m}^{2}$. This observation is true for every size of the exit sign. When visibility is expressed in terms of the visual angle (height of pictograph) it is independent of the size of the sign.

Figure 4 shows the relation between conspicuousness of ordinary type exit sign and the visual angle.[1] The larger size exit sign is more conspicuous than smaller size one when the visual angle is the same. This indicates that the conspicuousness depends on the relative scale of exit sign against the surrounding lights. In this sense, the visibility expressed in terms of visual angle does not always correspond rationally to conspicuousness.

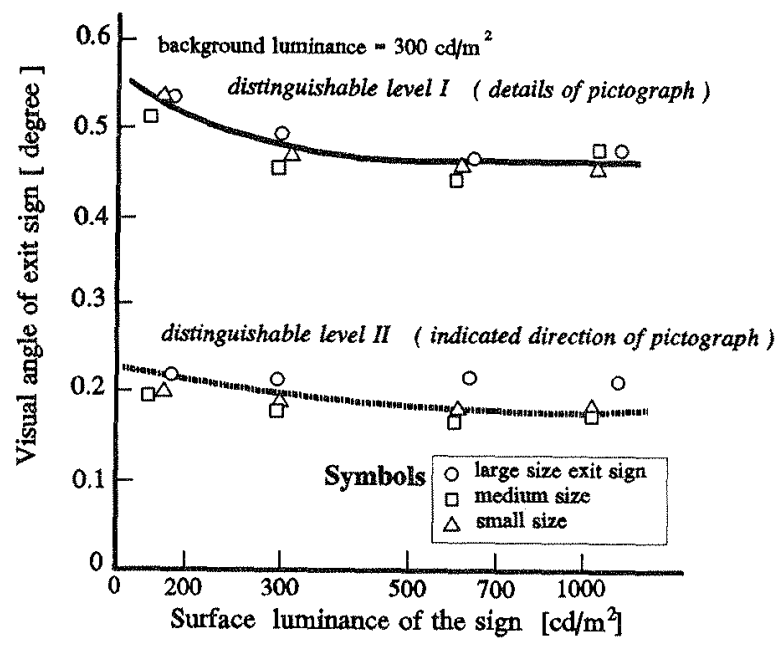

FIGURE 3. Relation between surface luminance of ordinary exit sign and visual angle as fa as visibility concerns.

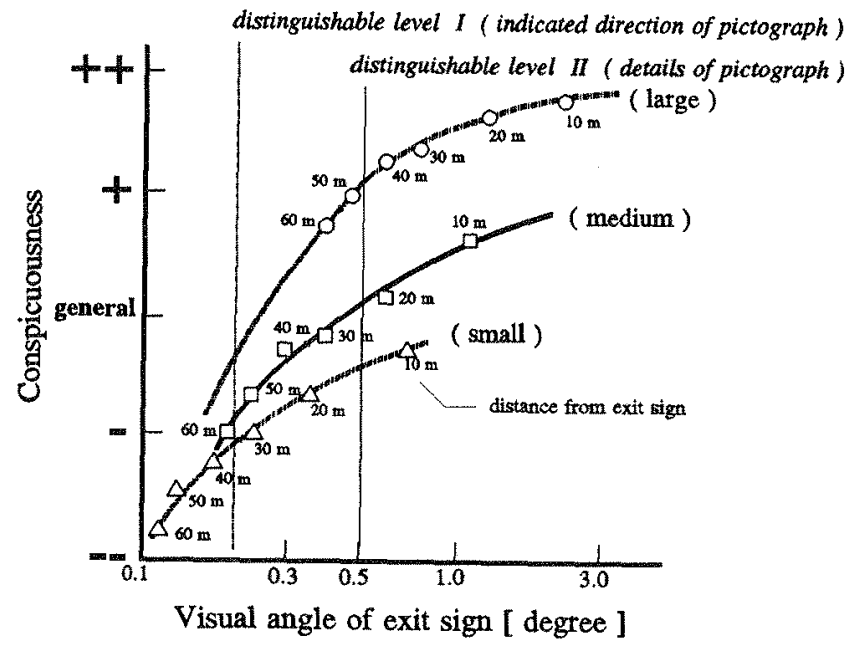

FIGURE 4. Relation between conspicuousness of ordinary type exit sign and visual angle. 


\section{Contrast of Luminance and Size}

For each size of sign, we get the relation between observation distance and luminance of signs when the evaluation category "conspicuousness - general level" is obtained, from Figure 2 and other data. Figure 5 shows that the relation between contrast of luminance [luminance of exit sign/adaptation luminance] and apparent size [(area of sign surface) /(observation distance $\left.)^{2}\right]$.

The adaptation luminance is about $150 \mathrm{~cd} / \mathrm{m}^{2}$ in the worst condition for finding out the exit sign (condition with many lighting sign boards and Neon-signs), $30-35 \mathrm{~cd} / \mathrm{m}^{2}$ in the room of normal brightness and $0 \mathrm{~cd} / \mathrm{m}^{2}$ in completely dark place. The adaptation luminance of background in the situation of the underground shopping plaza experiment is estimated to be $70-80 \mathrm{~cd} / \mathrm{m}^{2}$.

In Figure 5, the line of [ (contrast of luminance) $\times$ (visual angle) $=500]$ is shown. The line of the large size sign is almost parallel to this line. This fact means that when the observation distance is shortened to half (visual angle becomes double), the luminance of the exit sign can be reduced to half, to keep the conspicuousness the same. On the other hand the gradients of the line of the medium and small exit signs in the figure are less steep. Therefore, to keep the conspicuousness the same, their luminance cannot be reduced so much, even when observed from a nearer spot.

The reason why the large size exit sign is conspicuous even with the low luminance is that its size is sufficiently large compared with the size of the competing light sources. The large size exit sign is more conspicuous than the medium size one when both are observed at the position where the visual angle are just the same (observation distance of medium size exit sign is half of the distance of the large one because of the difference of size).

Consequently, the conspicuousness of the exit sign when surrounded with the light noises related deeply to the size of the sign itself. This fact suggests that the large size exit sign is very effective in such circumstance where there are many competing light sources as in an underground shopping plaza.

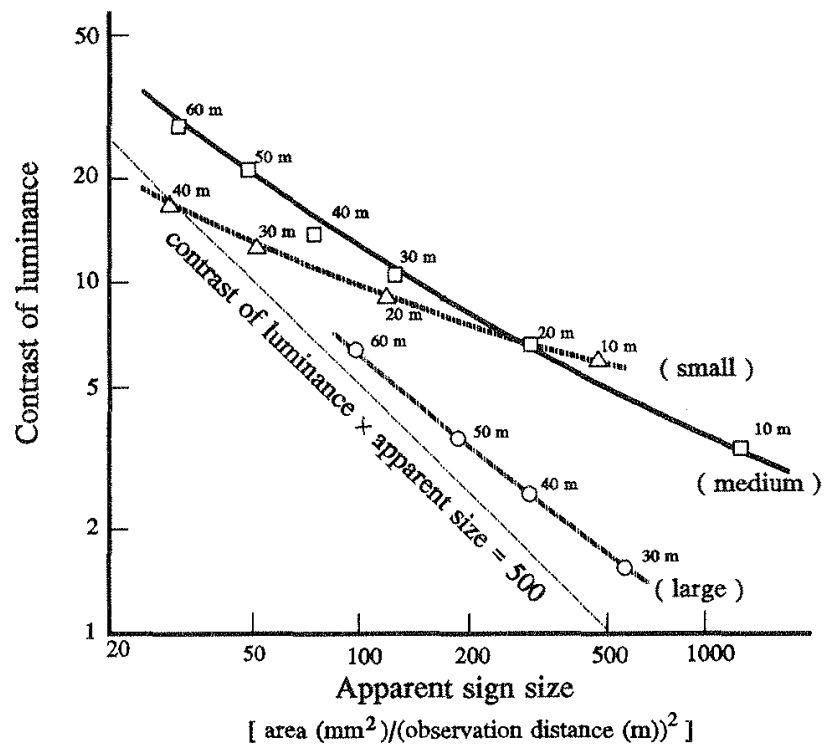

FIGURE 5. Relation between apparent sign size and contrast of luminance: [ luminance of exit sign / adaptation luminance ] 


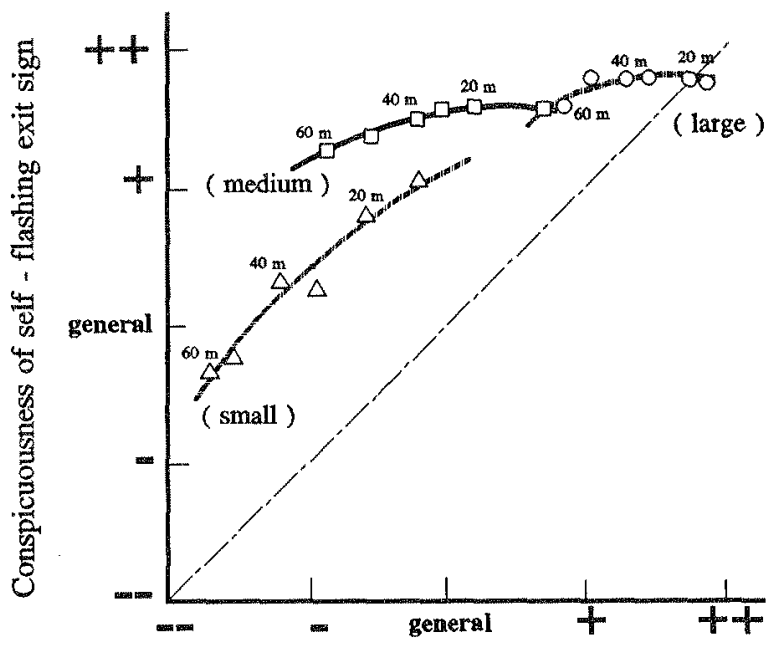

Cospicuousness of ordinary exit sign

FIGURE 6. Relation between conspicuousness of self-flashing exit sign and that of ordinary one.

\section{IMPROVEMENT OF CONSPICUOUSNESS BY FLASHING THE LIGHT SOURCE}

Figure 6 shows the relation between conspicuousness of ordinary type exit sign and that of the self-flashing type exit sign. In this figure the vertical interval between the dash-dotted line and the curves corresponds to the improvement of conspicuousness by the flashing light in the sign.

As for the large exit sign, the curve is closed to the dash-dotted line. It is because the large nonflashing exit sign itself is sufficiently conspicuous within $60 \mathrm{~m}$ observation distance. On the contrary, for the medium size exit sign, flashing the light is more effective to improve conspicuousness. The small size exit sign with flashing light is not conspicuous enough, even though the flashing light is supposed to be much conspicuous. This indicates the exit sign is too small to be recognized as an exit sign.

\section{CONCLUSION}

The conspicuousness of the exit sign in a background with many other light sources depends on both its size and luminance. This conspicuousness can be improved by flashing the light in the exit sign, and a degree of the improvement is dependent of the size of the exit sign. The large exit sign is big enough to have sufficient conspicuousness without flashing. On the other hand, the small exit sign is too small to be recognized as an exit sign even with flashing. The self-flashing medium sized exit sign improves conspicuousness even in the background with many competing light sources.

\section{REFERENCE}

1. Illuminating Enginecring Institute of Japan, The Report of Basic Research on Visibility of Exit Sign (1), 1984 
